\title{
Intelligent water meter with low power consumption based on ZigBee technology
}

\author{
Zhe Xie Rangding Wang ${ }^{1}$
}

\begin{abstract}
A design of intelligent water meter with low power consumption is present in this paper. MSP430 MCU and ZigBee module are applied in this design. The management of the water meter and the communication between ZigBee network and family gateway are completed at the same time. A node with low power consumption which can work for a long time in the network mode is developed. The problem which timing meter reading system can not be timely communication is resolved in this design. Finally, the experimental results demonstrate the feasibility of the proposed scheme.
\end{abstract}

Keywords: Water meter · ZigBee MSP430 $\cdot$ Low power consumption.

\section{Introduction}

With the update of the various types of meter reading system, its research and development is surrounded by the mode of meter reading, the accuracy of measurement and low power consumption. The household water meter has been going through two stages. The common mechanical water meter has been replaced by the ultrasonic water meter with high precision and low power consumption [1]. The centralized community of meter reading has taken the place of the traditional manual meter reading. Nowadays, the technology of remote wireless meter reading has made rapid progress. Although some intelligent remote water meter has been existed in the market, the precision of them is low and the power consumption is big. In addition, as for a timing meter reading system which has been improved in power consumption, but the requirement of real-time communication

Rangding Wang $(\varangle)$

College of Information Science and Engineering, Ningbo University, Ningbo, China

wangrangding@nbu.edu.cn 
can't be fully satisfied. So far, the wireless meter reading specification has not been unified. Large-scale application can't be realized in residential area, most of them are just a pilot. The popularization and application of wireless meter reading system hindered by the power consumption. The main reasons are the total power only relies on battery and the integration of wireless communication is difficult [26]. Thus, Wireless intelligent water meter with low power consumption, high cost performance, real-time communication research has realistic significance.

In this paper, The intelligent meter reading mode is based on the family gateway [7], and the water meter focuses on low power design. Some functions have come true. Realization 1:Communication between node and gateway's ZigBee network, 2: The sleep mode of system network, 3: The design of low power consumption to wake up on-demand [8]. Finally, an intelligent water meter with low power consumption based on ZigBee technology is developed.

\section{Intelligent meter reading mode}

The CPU of the intelligent water meter uses 430 Single Chip(Most of the water meter manufacturers use MSP430 on the market) , besides, ZigBee RF module is embedded in the water meter, the CPU controls the CC2520 RF through the SPI port. The reading company visits home gateway through the Internet to read meter's data in real-time. The diagram of the framework shows below:

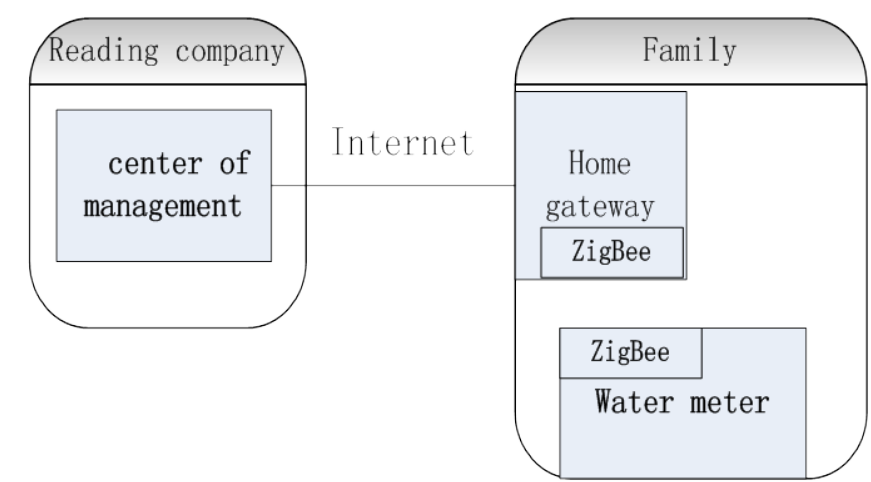

Fig.1 The framework of Meter reading mode

This model greatly reduces the manpower and material resources. At the same time, it makes the household water meter into the Internet of things and speeds up the process of intelligent home furnishing $[9,10]$. Problems and key technologies to solve the popularization of this new type of meter reading mode is the need to design a water meter with low power. In this paper, the hardware circuit optimization and the protocol of the software are designed to realize the low power consumption. 


\section{Design of hardware circuit}

The circuit designed by 4 lines SPI: CSN, SCK, MISO and MOSI Control the RF module. The serial input and output lines MISO/MOSI Control Data transmission; Rising edge of a pulse comes from the serial clock line SCK; The CSN receiver chip select signal from the 430 processor; At the same time, The Position of VREG_EN's voltage play an important role in the RF power [11]. MSP430F5437 and CC2520 hardware connection diagram shows below:

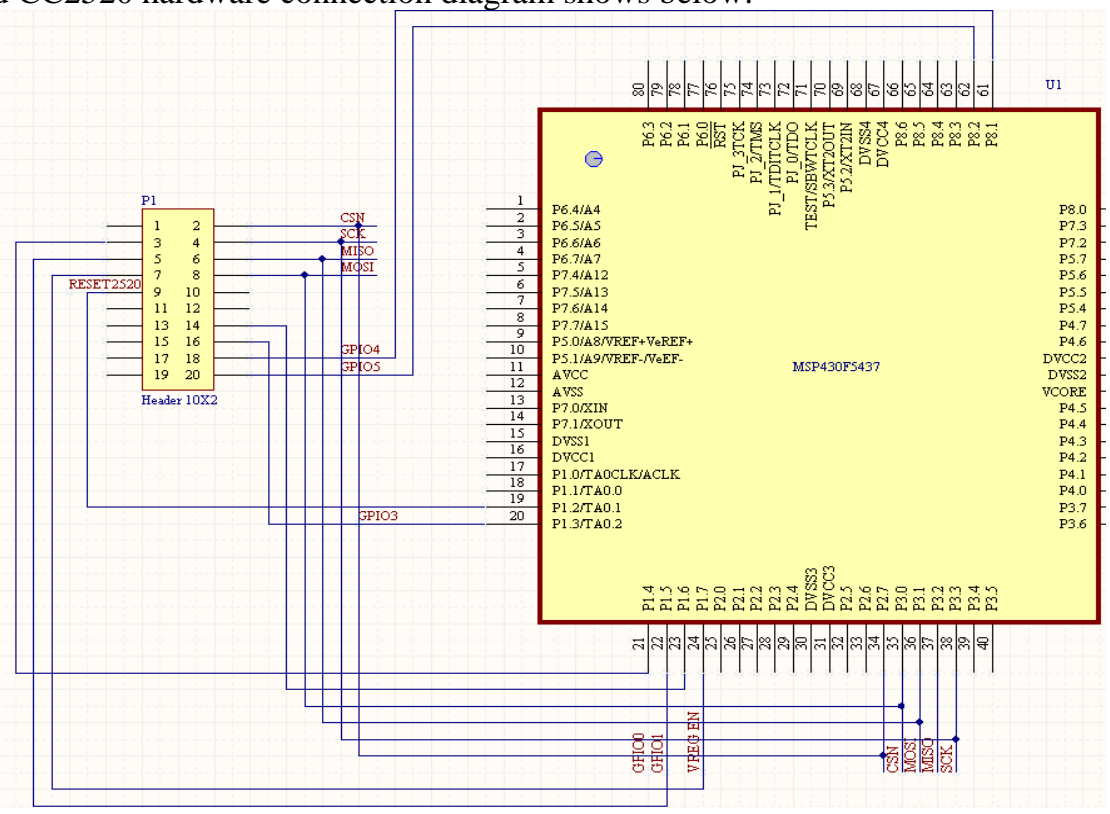

Fig.2 Processor and RF module connection diagram

\section{Design of software for low power}

Consumption is mainly reflected in two aspects: CPU and RF chip power consumption. In order to reduce the consumption, Light sleep mode wakes for the ondemand are the core of low power design.

\subsection{CPU power consumption}

Microcontroller 430 has 5 low power modes: LPM0 4, LPM0 closed CPU and MCLK; LPM1 close the CPU, MCLK, DCO; LPM2 close the CPU, MCLK, 
SMCLK, DCO; LPM3 close the CPU, MCLK, SMCLK, DCO, DC; LPM4 close the CPU and all clocks.

At different operating frequencies, power consumption is different, Working power and standby power consumption of MSP430F5437 are 160uA/MHZ and 1.5uA/MHZ. The CPU chooses different operating frequencies in a different time (As in the time of data collection, in order to improve the precision of data acquisition, the frequency is set to $8 \mathrm{MHZ}$, this power consumption is relatively large). The appropriate working voltage is selected to ensure the normal work. This paper provides working voltage of $3.0 \mathrm{~V}$ to the processor through the MC78PC30 power conversion chip, it can reduce power consumption current. The dormancy of low power design the processor which Z-stack combines LPM3: In the HAL layer of Z-stack (\#define HAL_SLEEP_TIMER), in a non-operating state, the water meter goes to mild sleep mode. Until the RF module receives a meter reading command, the CPU is waked up to collect data.

\subsection{RF module power consumption}

For RF module, transmit power is greater, the transmission distance is longer, module power consumption is bigger. In this contradiction, according to the actual distance, we select transmit power properly to make the communication distance and power tradeoff. Transceiver current as shown in Table 1: The RF power is visible large, this paper reduces the power consumption by shortening the sending and receiving time.

Table 1: CC2520 current in the receiving and sending

\begin{tabular}{ll}
\hline \multicolumn{2}{c}{ Current consumption } \\
\hline Current consumption, RX & $22 \mathrm{~mA}$ \\
Current consumption, TX, $+5 \mathrm{dBm}$ & $33 \mathrm{~mA}$ \\
Current consumption, 0dBm & $25 \mathrm{~mA}$
\end{tabular}

At the same time, this paper studies that CC2520 wakes up the CPU (ondemand wakeup function) which guarantees the real-time meter data acquisition

\subsection{Light-sleep mode}

When the device enters sleep mode, in order to prevent the disconnection of water meter and the home gateway, the timer is started (light sleep mode of TIMER_SLEEP uses a $32.768 \mathrm{KHZ}$ crystal to drive timers work) : Ticks = halSleepTimerStart; According to the equipment, The ticks determine the time by the crystal oscillator: $32.768 \mathrm{khz} / 8=4096 \mathrm{hz}$. 
Calculation formula:

Ticks += (timeout $* 4096$ / 3125);

When the timeout reaches the upper limit, the system automatically wakes up. So, The CPU control CC2520 to send a connection information to the home gateway. In this way, it ensures that the water meter is in the ZigBee network. Increasing the timing time as long as possible to make the water meter stay in low-power sleep mode to reduce power consumption.

\subsection{Interrupt for on-demand wake up}

To prevent the system from missing gateway in the sleep mode during the reading order, this paper design to keep the interrupt opening in TIMER_SLEEP mode. The node stays in the Network state, an interrupt (HAL_SLEEP_TIMER_ENABLE_INT) is generated so that the CPU will be waked to read meter data. Finally, it completes the wireless transmit operation.

The low power design flow chart of the water meter node is shown as follows:

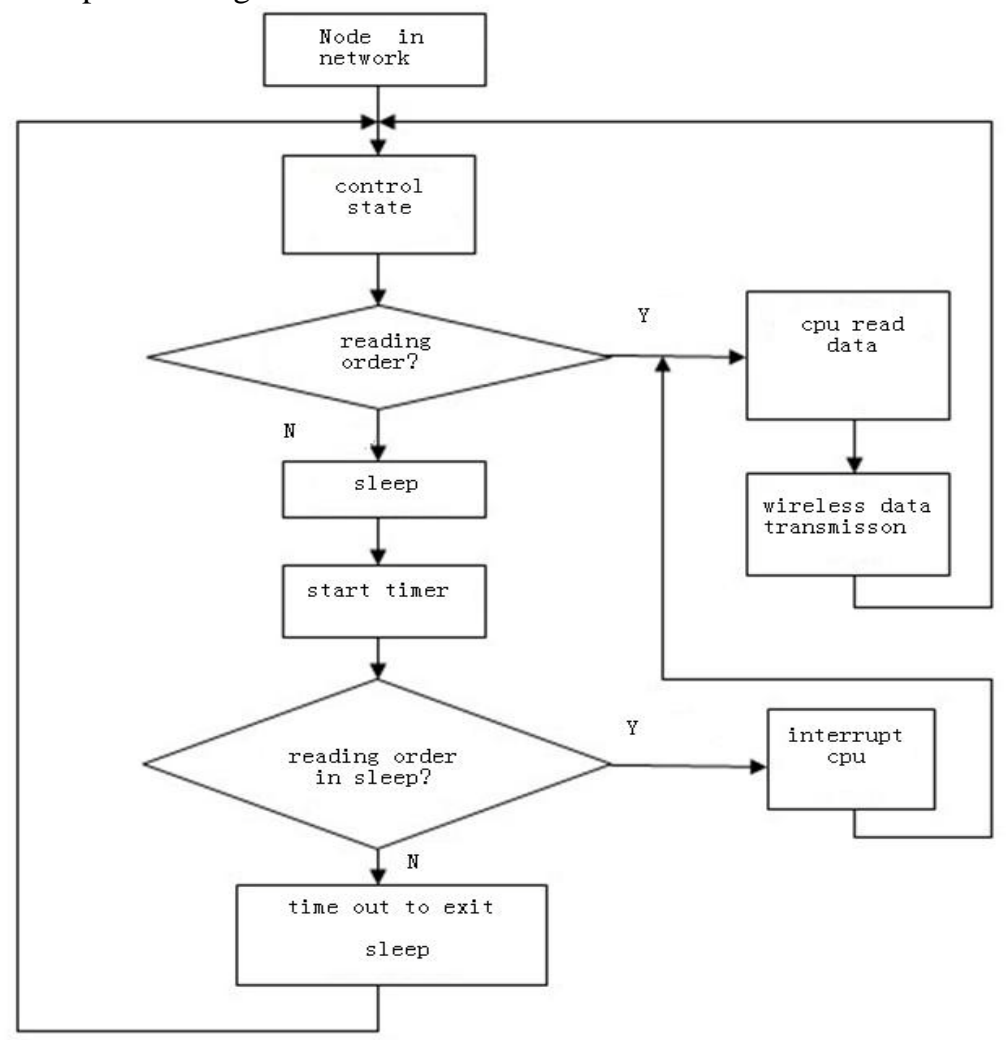

Fig.3 The flow chart of low power design 


\section{Power test}

The water meter goes to sleep mode which can extend battery life. A decisive factor of the power size is a long time sleep mode in the network state. An experiment about the power test has been done in this paper. The experimental platform is based on the high performance's 3 regulated power supply, the high performance digital current meter and oscilloscope. Test experimental data for some time shows below:
$\mathrm{P} 1=\mathrm{U} * \mathrm{I}=3.0 \mathrm{v} * 1.943 \mathrm{~mA}=5.829 \mathrm{mw}$;
$\mathrm{P} 2=\mathrm{U} * \mathrm{I}=3.0 \mathrm{v}^{*} 0.089 \mathrm{MA}=0.267 \mathrm{mw}$
$\mathrm{Pc}=\mathrm{P} 1+\mathrm{P} 2=6.096 \mathrm{mw}$;

P1: The power of RF module in sleep, P2: The power of CPU in sleep mode, Pc: The total power of the water meter. The figure 4 shows the power current meter in the sleep state in 5 minutes. The fundamental wave is $2.1 \mathrm{~mA}$ nearby from the picture.

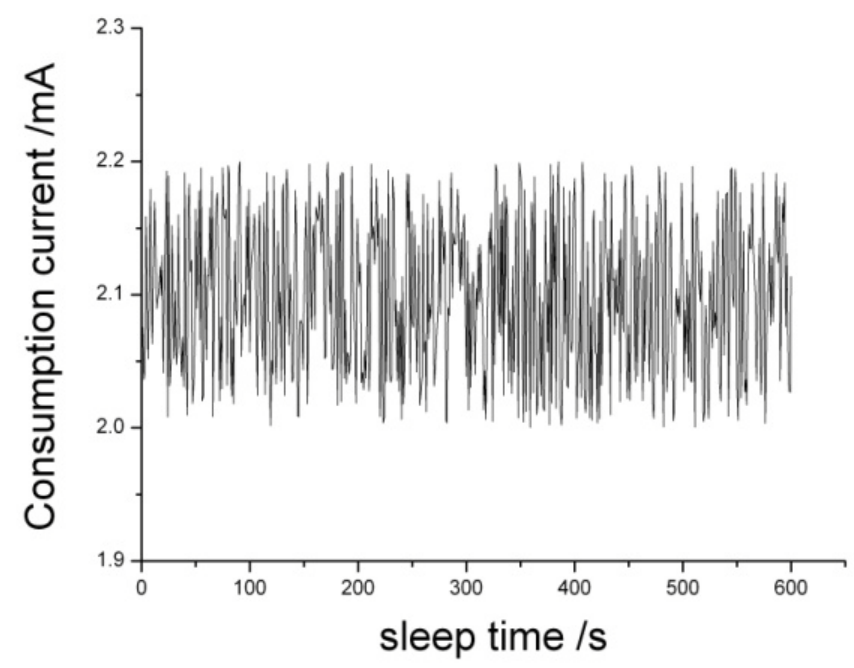

Fig.4 Consumption current in the sleep time

\section{Conclusion}

Providing a battery with voltage 3.6V, capacity of $19000 \mathrm{MAH}$ to calculate the life of water meter.

The battery life calculates: 


$$
t=\frac{3.6 V^{*} 19 \times 10^{3} \mathrm{~mA} \cdot \mathrm{h}}{6.096 \mathrm{mw} \times(24 \times 365)}=1.28 y e a r
$$

Visibly, the replacement time of battery life is approximately a year. Compared with the mechanical water meter which has no wireless reading function, battery life has been reduced in a certain degree, but real-time wireless communication technology of the intelligent water meter with low power has great application value. Water meter node displays as fig 5 .

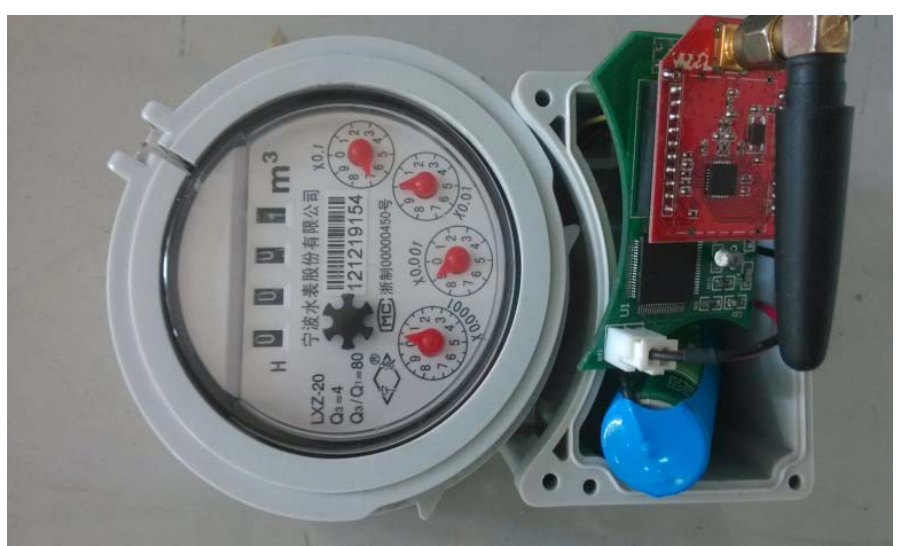

Fig.5 Water meter display

Acknowledgements This work is supported by the National Natural Science Foundation of China (NSFC: 61170137), Doctoral Fund of Ministry of Education of China (20103305110002), Zhejiang science \& technology preferred projects of China (2010C11025), Zhejiang province education department key project of China (ZD2009012), Ningbo key service professional education project of China (2010A610115)

\section{References}

1. Qiang Liu, Rangding Wang, Ling Yao(2012). Research on low power design methods of time difference ultrasonic flow meter. Journal of Ningbo University. 25(2):1001-5132.

2. Subing Shen, Yanqin Mao(2010). Probability model and the architecture of the Internet of things. Journal of Nanjing University of Post and Telecommunication. 04:1673-5439.

3. Yinkang Chen(2008). Concentrator hardware design of the wireless intelligent meter reading system. Components and IC:1003-0107. 
4. Li Cao, Huanglin Zeng(2012). Based on ZigBee and MSP430‘s wireless temperature control system design. Journal of Sichuan Polytechnic. 25(1):1673-1549.

5. Huajian Wang, Lihua Zhang(2010). Design of wireless meter reading terminal based on MSP430 and ZigBee. Design and application of electronic technology. 37(12):1000-0755.

6. Huifeng Zhu, Qiang Ding(2009). Low power RTU design based on MSP430 single chip microcomputer. Water conservancy hydrology automation. (1):1674-9405.

7. Yifeng Zhang(2012). Research and implementation of intelligent home gateway based on the integration of three networks. Yanshan University. (1):1467-3490.

8. Weibin Bao, Weijie Chen(2013). Design and implementation of wireless meter reading system based on ZigBee. Industrial instrumentation and automation device. (02):1000-0682.

9. Shoufeng Wang, Guoli Lin(2012). Application of wireless remote meter reading system based on GSM. Management informatization in China. (04):1673-0194.

10. Jun Chen, Jiaping Deng(2010). Research on intelligent electric meter reading system based on Wireless Sensor Network. Electrical measurement and instrumentation. (08):1001-1390.

11. Ming Liu(2013). Low power design of intelligent temperature alarm system. Science and technology innovation. (02): 1673-9795. 